\title{
Supplementary Material for
}

Application of a GC-ECD for measurements of biosphere-atmosphere exchange fluxes of peroxyacetyl nitrate using the relaxed eddy accumulation and gradient method

\author{
A. Moravek, T. Foken and I. Trebs \\ Correspondence to: A. Moravek (a.moravek@mpic.de) \\ published in Atmospheric Measurement Techniques Discussions 2014
}

\section{Contents}

\section{SM 1 Operation of PAN GC}

SM 1.1 Main settings

SM 1.2 Design and operation of PCUs

SM 2 Fast response system design for HREA operation

SM 2.1 Time response of data transmission, HREA software and splitter valve actuation

SM 2.2 Time response of splitter valves

SM 3 Calculation of random errors using Gaussian error propagation

SM 4 Correction method for PAN mixing ratio differences during HREA operation

SM 5 Additional figure

References 


\section{SM 1 Operation of PAN GC}

\section{SM 1.1 Main settings}

During the operation of the GC-ECD the temperature of the Peltier-cooled oven module, which houses both columns, was set to $18^{\circ} \mathrm{C}$ to prevent thermal decomposition of PAN at higher temperatures. For the same reason, the ECD was heated to only $50^{\circ} \mathrm{C}$. We used nitrogen 5.0 (Air Liquide Deutschland GmbH, Germany) as both carrier gas (volume flow $18 \mathrm{~mL} \mathrm{~min}^{-1}$ ) and make up gas for the ECD (volume flow $16 \mathrm{~mL} \mathrm{~min}^{-1}$ ). The chosen carrier gas flow rate gave the best compromise to separate the PAN peak (PAN retention time $=243 \mathrm{~s}$ ) from carbon tetrachloride $\left(\mathrm{CCl}_{4}\right.$, retention time $\left.=203 \mathrm{~s}\right)$ and to minimize the total analysing time. To prevent changes in humidity levels between carrier gas and sample air, the carrier gas was humidified in the GC-ECD by flowing through a thermally-controlled cartridge filled with $\mathrm{CuSO}_{4} \cdot 6 \mathrm{H}_{2} \mathrm{O}$ prior to injection (Flocke et al., 2005).

\section{SM 1.2 Design and operation of PCUs}

The PCUs consist of a $1.15 \mathrm{~m}$ capillary column (MXT-1, ID $0.53 \mathrm{~mm}$, film thickness $7.00 \mu \mathrm{m}$; .Restek, USA) as a trapping material, which is coiled in an insulated circular aluminium (ID $35 \mathrm{~mm}$ ) housing and cooled by two peltiers (Supercool PE-127-08-25-S, Laird technologies) arranged in series. To optimize the stability of the temperature control, the inner space of the housing was filled by an aluminium cylinder, which could be removed when the column had to be replaced. The temperature of the aluminium housing was monitored by a Pt100 probe and regulated to $-5^{\circ} \mathrm{C}$. For injections, the capillary column is heated by passing a current through the column using a $12 \mathrm{~V} \mathrm{DC}$ supply. To prevent a short circuit the MXT-column $(R=\sim 4.5 \Omega)$ was coated with 1/16" PFA tubing. The heating of the column was limited to $25^{\circ} \mathrm{C}$ to prevent thermal decomposition of PAN at higher temperatures, while it was assured that the coil was heated long enough to allow slow substances to evaporate from the column. A balance between the maximum heating temperature, the heating time and the cooling temperature of the aluminium housing had to be found as typically the column temperature declined slowly after heating and had to reach the initial temperature of $-5^{\circ} \mathrm{C}$ before switching to the sampling mode (after $5 \mathrm{~min}$ ). 


\section{SM 2 Fast response system design for HREA operation}

\section{SM 2.1 Time response of data transmission, HREA software and splitter valve actuation}

The PC housing the HREA software program (LabVIEW, National Instruments Corporation, USA) was connected via RS232 with the CR3000 data logger (see Fig. 1a in main article) to receive the high frequency $(20 \mathrm{~Hz})$ wind vector, the $\mathrm{O}_{3}$ signal for calculation of the hyperbolic dead band and the determination of updraft and downdraft events. The signal of the in-built closed-path $\mathrm{CO}_{2}$ analyser was transmitted for the lag time determination (see Sect. 2.4 in main arcticle). The data was transmitted at a baud rate of $57.6 \mathrm{kBit} \mathrm{s}^{-1}$ using the four byte floating point (IEEE4) data type which resulted in a time span between the start and end of the data string of about $10 \mathrm{~ms}$. A higher baud rate could not be used due to the length $(\sim 20 \mathrm{~m})$ of the RS232 data cable. To assure an accurate timing of the HREA software on the PC, the execution interval $(50 \mathrm{~ms})$ was triggered by the incoming string sent from the CR3000. The splitter valves were actuated using a PCI-DAQ card (National Instruments Corporation, USA). To avoid any delays caused by the PC's operation system (Windows XP, Microsoft, USA), the PC's system preferences had to be set to best performance. The elapsed time between the sending of the signal from the CR3000 until the actuation of the splitter valves was measured to be less than $20 \mathrm{~ms}$.

\section{SM 2.2 Time response of splitter valves}

The time response of the splitter valve was tested by recording the signal of a fast response pressure sensor (HCX series, Sensortechnics $\mathrm{GmbH}$, Germany) mounted in a miniature inlet system (Fig. 1) while the valve was opened and closed with different frequencies. The results are shown in Fig. 2 for switching frequencies of 10, 20, 33 and $100 \mathrm{~Hz}$. Whereas for 10, 20 and $30 \mathrm{~Hz}$ the pressure signal still follows the step function of the valve switching, at $100 \mathrm{~Hz}$ clear deviations are visible. 


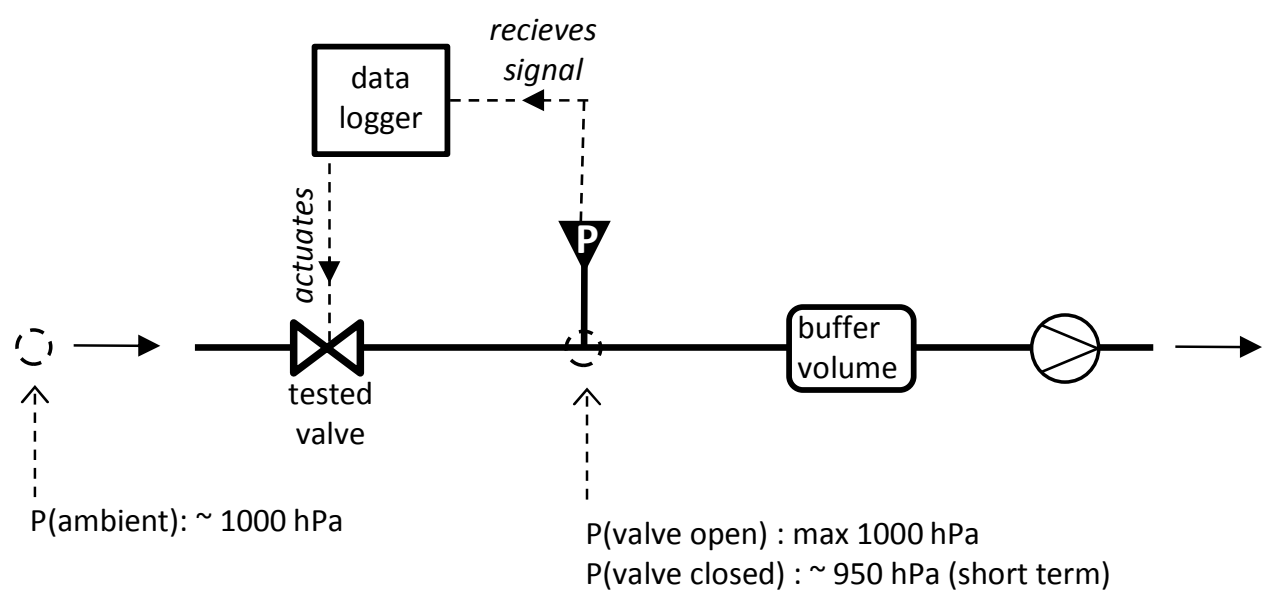

Fig. 1. Setup used to test the response time of the splitter valves. A data logger actuates the splitter valve with a certain frequency and records the pressure variation in a miniature inlet tube, induced by the opening and closing of the valve.

(a)

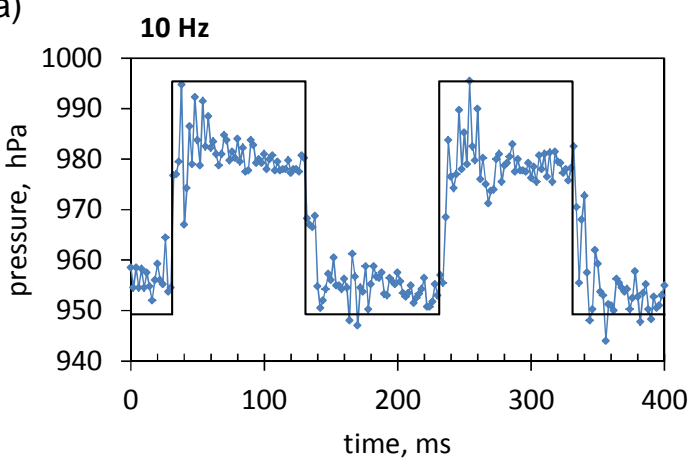

(c)

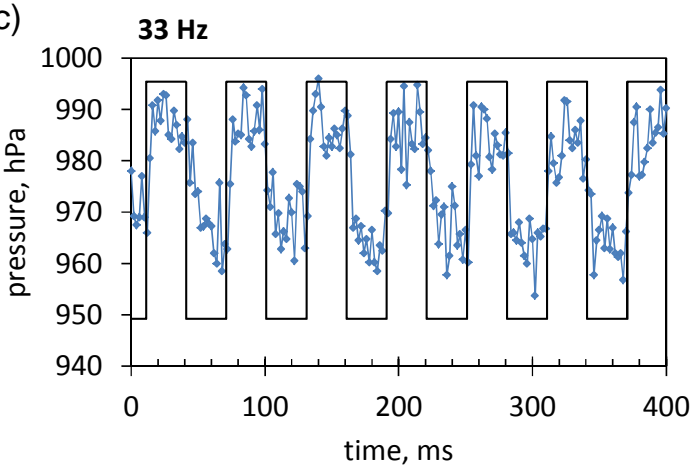

(b)

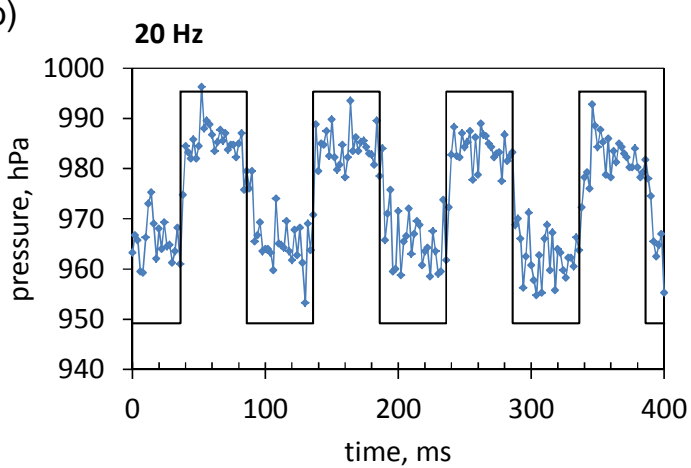

(d)

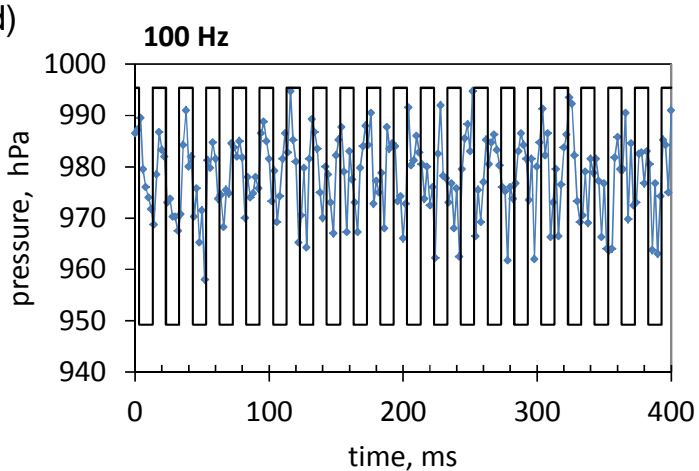

Fig. 2. Results of the response time test of the used splitter valves. (a-d) Shown are the pressure variations induced by switching frequencies of $10,20,33$ and $100 \mathrm{~Hz}$. 


\section{SM 3 Calculation of random errors using Gaussian error propagation}

The general form of the Gaussian error propagation equation was used to determine (a) the random flux errors, (b) the random errors of $\Delta \chi_{O_{3}(M B R)}$ and (c) the random errors of the $b$ value. The variance of a function $(f)$ with $n$ individual quantities $\left(x_{i, j}\right)$ is described as (e.g., Bevington and Robinson, 2003):

$$
\begin{aligned}
\sigma_{f}^{2}= & \sum_{i=1}^{n} \sigma_{x_{i}}^{2}\left(\frac{\partial f}{\partial x_{i}}\right)^{2} \\
& +\sum_{i=1}^{n} \sum_{j=1}^{n}\left(\left(\frac{\partial f}{\partial x_{i}}\right)\left(\frac{\partial f}{\partial x_{j}}\right) \sigma_{x_{i}} \sigma_{x_{j}} r\left(x_{i}, x_{j}\right)\right), \quad i \neq j
\end{aligned}
$$

where $\sigma_{x_{i, i}}$ denotes the random error of the individual quantities (see Sect. 2.6.3 in main text for their determination) and $r$ the correlation coefficient between two dependent quantities $\left(x_{i}, x_{j}\right)$. In case the quantities are uncorrelated the covariance term $\left(\sigma_{x_{i}} \sigma_{x_{j}} r\left(x_{i}, x_{j}\right)\right)$ is zero. After solving the partial derivatives of $f$ and substituting those in Eq. (1), the random error of an individual estimate of $f$ is determined by the square root of $\sigma_{f}^{2}$. For the errors (a) - (c) $f$ is defined as:

(a) for the random error of HREA flux:

$$
f_{H R E A}:=F_{P A N}=\frac{F_{O_{3}}}{\Delta \chi_{O_{3}(H R E A)}} \cdot \Delta \chi_{P A N}
$$

and accordingly for the MBR flux:

$$
f_{M B R}:=F_{P A N}=\frac{F_{O_{3}}}{\Delta \chi_{O_{3}(M B R)}} \cdot \Delta \chi_{P A N}
$$

(b) for the random error of $\Delta \chi_{O_{3}(M B R)}$ :

$$
f_{\Delta \chi_{O_{3}(M B R)}}:=\Delta \chi_{O_{3}(M B R)}=\Delta S_{O_{3}}(\text { ambient })-\Delta S_{O_{3}}(0)
$$

where $\Delta S_{O_{3}}$ (ambient) and $\Delta S_{O_{3}}(0)$ are the calibrated differential signals at ambient and zero air measurements.

(c) Finally, for the random error of the $b$ value: 


$$
f_{b}:=b=\frac{\overline{w^{\prime} \chi_{O_{3}}{ }^{\prime}}}{\sigma_{w} \cdot \Delta \chi_{O_{3}(H R E A)}}
$$

where $\overline{w^{\prime} \chi_{O_{3}}{ }^{\prime}}$ is the covariance between the vertical wind velocity $(w)$ and the $\mathrm{O}_{3}$ mixing ratios measured by the fast $\mathrm{O}_{3}$ analyser. The error of $\sigma_{w}$, which is needed for the derivation of $\sigma_{b}$ (see Eq. (1)), was determined using the simplified version of $\mathrm{Eq}$, (1) without the covariance term. After expanding the general form of the standard deviation:

$$
\sigma_{w}=\sqrt{\frac{1}{m-1} \sum_{i=1}^{m}\left(w_{i}-\bar{w}\right)^{2}}
$$

we obtain the partial derivative $\left(\frac{\partial \sigma_{w}}{\partial w_{i}}\right)$ needed in Eq. (1):

$$
\frac{\partial \sigma_{w}}{\partial w_{i}}=\frac{w_{i} \cdot(1-\bar{w})}{\sqrt{\sigma_{w}} \cdot(m-1)}
$$

where $m$ is the number of measurements per averaging period (i.e. $m=36000$ for $30 \mathrm{~min}$ at a sample frequency of $20 \mathrm{~Hz}$ ). Finally, we obtain for the error of $\sigma_{w}$

$$
\sigma_{\sigma_{w}}= \pm \frac{s_{w} \cdot(1-\bar{w})}{\sqrt{\sigma_{w}} \cdot(m-1)} \sqrt{\sum_{i=1}^{m} w_{i}^{2}}
$$

where $s_{w}$ is the random error of a single measurement of $w$ (given by the manufacturer as $0.5 \mathrm{~mm} \mathrm{~s}^{-1}$, see Sect. 2.6.3 in main text).

The values for the correlation coefficient between the individual quantities are given in Table 1 for the HREA measurement period (for determination of $\sigma_{F_{P A N}}$ and $\sigma_{b}$ ) and Table 2 for the MBR measurement period (for determination of $\sigma_{F_{P A N}}$ ) separated for daytime and nighttime. The covariance term was not considered for the determination of $\sigma_{\triangle \chi_{O_{3}(M B R)}}$ as the correlation between $\Delta S_{O_{3}}$ (ambient) and $\Delta S_{O_{3}}(O)$ was not significant during both daytime $(r=-0.01)$ and nighttime $(r=-0.02)$. 
Table 1. Matrix of correlation coefficients $(r)$ between dependent quantities $\left(x_{i}, x_{j}\right)$ over the period of the HREA measurements for daytime/nighttime conditions.

\begin{tabular}{rcccc}
\hline & $\Delta \chi_{P A N}$ & $\Delta \chi_{O_{3}(H R E A)}$ & $F_{O_{3}}$ & $\sigma_{w}$ \\
\hline$\Delta \chi_{P A N}$ & 1 & $0.21 / 0.57$ & $-0.02 / 0.48$ & - \\
$\Delta \chi_{O_{3}(H R E A)}$ & - & 1 & $0.18 / 0.42$ & $-0.25 /-0.45$ \\
$F_{O_{3}}$ & - & - & 1 & $-0.63 /-0.66$ \\
$\sigma_{w}$ & - & - & - & 1 \\
\hline
\end{tabular}

Table 2. Matrix of correlation coefficients $(r)$ between dependent quantities $\left(x_{i}, x_{j}\right)$ over the period of the MBR measurements for daytime/nighttime conditions.

\begin{tabular}{rccc}
\hline & $\Delta \chi_{P A N}$ & $\Delta \chi_{O_{3}(M B R)}$ & $F_{O_{3}}$ \\
\hline$\Delta \chi_{P A N}$ & 1 & $0.21 / 0.57$ & $-0.02 / 0.48$ \\
$\Delta \chi_{O_{3}(M B R)}$ & - & 1 & $0.18 / 0.42$ \\
$F_{O_{3}}$ & - & - & 1 \\
\hline
\end{tabular}




\section{SM 4 Correction method for PAN mixing ratio differences during HREA operation}

As demonstrated in the main article, the scatter during HREA operation was significantly larger, which suggests that pressure differences induced by the fast switching of the splitter valves or varying sample volumes may influence the quality of the PAN measurement. Indeed, we found a linear relationship between the ratio of the normalized PAN integrals measured by PCU\#1 and PCU\#2 and the difference between the sampled gas volumes of both PCUs $(\Delta v o l=v o l \# 1-$ vol\#2) (Fig. 3). Using the slope $(m)$ of this relationship we could implement a correction of the normalized PAN integrals for PCU\#1:

$$
\left(\frac{i n t \# 1}{v o l \# 1}\right)_{\text {mod }}=\frac{i n t \# 1}{v o l \# 1}+\frac{1}{4} \Delta v o l \cdot m \cdot\left(\frac{i n t \# 1}{v o l \# 1}+\frac{i n t \# 2}{v o l \# 2}\right)
$$

and accordingly for PCU\#2:

$$
\left(\frac{i n t \# 2}{\text { vol\#2 }}\right)_{\bmod }=\frac{i n t \# 2}{\text { vol\#2 }}-\frac{1}{4} \Delta v o l \cdot m \cdot\left(\frac{i n t \# 1}{\text { vol\#1 }}+\frac{i n t \# 2}{\text { vol\#2 }}\right)
$$

Applying the correction for the HREA side-by-side measurements improved the precision by $50 \%$, to 17.9 and $26.1 \mathrm{ppt}$, respectively. Hence, this correction was applied to all data in the post-processing of the HREA measurements.

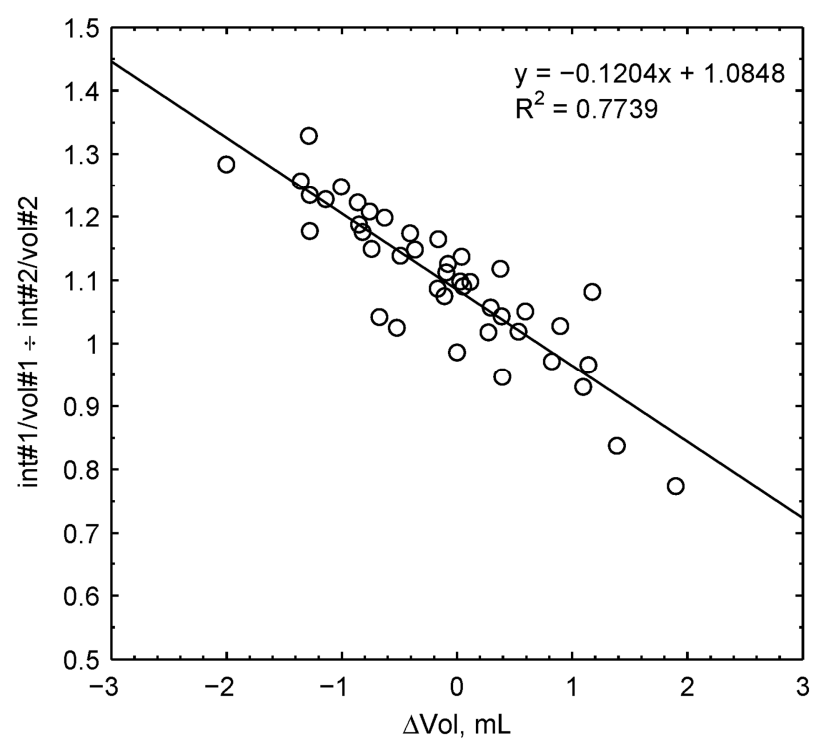

Fig. 3. Ratio of the normalized PAN integrals measured by PCU\#1 and PCU\#2 versus the difference between the sampled gas volumes of both PCUs $(\Delta v o l=v o l \# 1-v o l \# 2)$ during the HREA side-byside measurements (SBS_HREA\#2). The linear relationship was used to develop a correction function minimizing the random error of the HREA measurements. 


\section{SM 5 Additional figure}

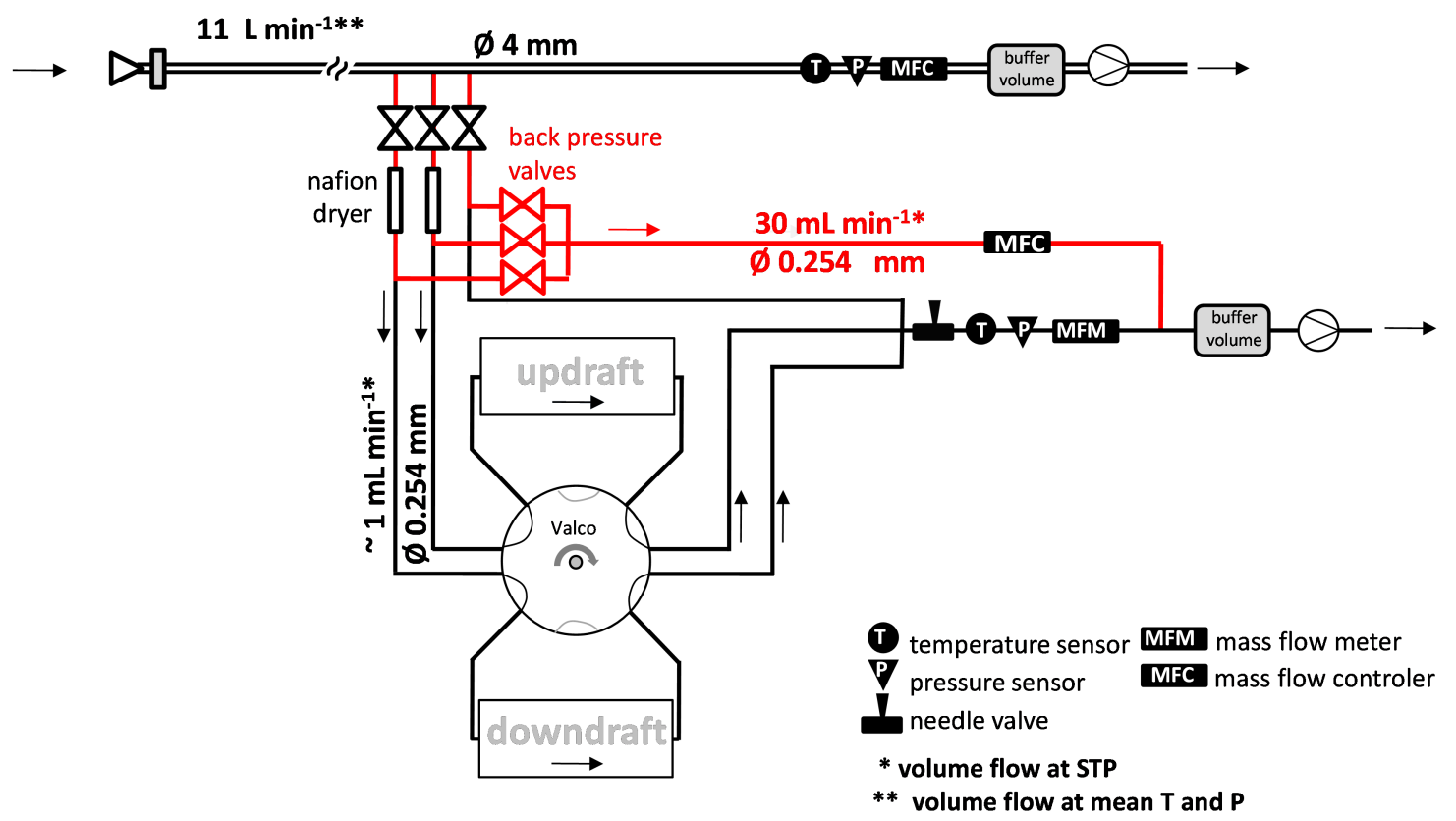

Fig. 4. Flow scheme of the PAN flux measurement system (shown in the HREA mode), highlighting the implementation of a bypass (marked in red) to enhance the flow rate through the splitter valves and the Nafion dryers. To prevent back-flushing of sample air into the wrong reservoir, three backpressure valves were employed, which were switched simultaneously with the respective splitter valve.

\section{References}

Bevington, P. R., and Robinson, D. K.: Data reduction and error analysis for the physical sciences, McGraw-Hill, 2003.

Flocke, F. M., Weinheimer, A. J., Swanson, A. L., Roberts, J. M., Schmitt, R., and Shertz, S.: On the measurement of PANs by gas chromatography and electron capture detection, J. Atmos. Chem., 52, 19-43, 10.1007/s10874-005-6772-0, 2005. 